\title{
Plasmonic nanoparticle heterodimers in a semiembedded geometry fabricated by stepwise upright assembly
}

\author{
Hui Wang and Naomi J. Halas* \\ Department of Chemistry, Department of Electrical and Computer Engineering, and the Laboratory for \\ Nanophotonics, Rice University, Houston, TX 77005 \\ *Corresponding author. Email: halas@rice.edu
}

Experimental details:

Tetraethyl orthosilicate (TEOS, 99.9999\%), (3-aminopropyl)trimethoxysilane (APTMS, >97\%), tetrachloroauric acid $\left(\mathrm{HAuCl}_{4} \cdot 3 \mathrm{H}_{2} \mathrm{O}\right)$, tetrakis hydroxymethyl phosphonium chloride (THPC, $80 \%$ solution in water), and poly(4-vinylpyridine) (160 000 MW) were purchased from Sigma-Aldrich (St. Louis, MO). Ammonium hydroxide and ethanol were obtained from Fisher Scientific (Hampton, NH). Silicone elastomer (PDMS, Sylgard 184) was purchased from Dow Corning Corporation. Ultrapure water (18.2 M $\Omega$ resistivity) was obtained from a Milli-Q water purification system (Millipore, Billerica, MA). Glass microscope slides were obtained from Gold Seal Products (Portsmouth, NH). All the chemicals were used as received without further purification. Extinction spectra were obtained using a Cary 5000 UV/Vis/NIR spectrophotometer. Scanning electron microscopy (SEM) measurements were performed using a JEOL 6500 scanning electron microscope. Atomic force microscopy (AFM) was performed with a Digital Instruments Nanoscope IIIa in tapping mode using a 3045JVW piezo tube scanner.

The fabrication of Au nanoshells has previously been described in detail. ${ }^{1}$ Briefly, colloidal silica nanospheres prepared by the Stöber method ${ }^{2}$ were used as the core material. The size of the silica cores was characterized by SEM and dynamic light scattering (DLS) measurements. The surface of the silica particles was then functionalized with APTMS, to generate an amine moiety-coated silica surface, and decorated with tetrakis hydroxymethyl phosphonium chloride stabilized Au nanoparticles ${ }^{3}$ ( $~ 2 \mathrm{~nm}$ in diameter). The attached Au colloids act as nucleation sites that catalyze the reduction of $\mathrm{AuCl}_{4}{ }^{-}$ions by formaldehyde, resulting in the formation of continuous $\mathrm{Au}$ nanoshells. The as-prepared nanoshells were washed through multiple steps of centrifugation and redispersion in Milli-Q water.

The as-fabricated nanoshells were immobilized onto poly(vinylpyridine)-functionalized glass substrates as a monolayer film of isolated nanoshells. ${ }^{4}$ Briefly, glass slides were treated in piranha solution (sulfuric acid : hydrogen peroxide, 7:3) and then immersed in a 1\% wt. solution of PVP in ethanol for 24 hours. The slides were rinsed thoroughly with ethanol and dried with $\mathrm{N}_{2}$ gas flow. Then the PVP-functionalized slides were immersed in an aqueous solution of nanoshells for 1 hour. Upon removal from the nanoshell solution, the films were rinsed with ethanol and dried with $\mathrm{N}_{2}$. This resulted in a monolayer of isolated nanoshells immobilized on the PVP functionalized glass surface. The surface of the immobilized nanoshells can be further functionalized with PVP by immersing the nanoshell films in 1\% wt. solution of PVP in ethanol for 12 hours. Then the films were removed from the PVP solution, rinsed thoroughly with ethanol and dried with $\mathrm{N}_{2}$. 
We used PDMS as the polymer mold to fabricate the partially embedded nanoshells. The PDMS was mixed with the curing agent (10:1 v/v) and outgassed under low pressure (100 milliTorr) at ambient temperature for half an hour before use. Then the PDMS was partially cured in an oven at $50{ }^{\circ} \mathrm{C}$ for $10 \mathrm{~min}$ to increase its viscosity. The partially cured PDMS was then poured over the monolaryer of PVP-functionalized nanoshells and further cured at $80{ }^{\circ} \mathrm{C}$ for 5 hours. Once cured, the PDMS can be peeled off the glass substrates, resulting in a PDMS slab with partially embedded PVP-functionalized nanoshells.

Nanoshell dimers can be fabricated upon the immersion of the embedded nanoshells in Au nanoshell solutions. Typically, we immersed a PDMS slab with partially embedded PVP-functionalized nanoshells in an aqueous solution of nanoshells $\left(\sim 10^{9}\right.$ particle $\left.\mathrm{mL}^{-1}\right)$ for $2 \mathrm{~h}$. Then the PDMS slab was removed from the nanoshell solution, rinsed with ethanol and water in sequence, and finally dried with $\mathrm{N}_{2}$. During this process, each nanoshell in solution can only be anchored exclusively to exposed surface on the top of individual embedded nanoshells. As a result, the as-fabricated nanoshell dimers have well-defined "standing-up" orientation with the dimer axis perpendicular to the PDMS slab surface.

\section{References}

(1) Oldenburg, S. J.; Averitt, R. D.; Westcott, S. L.; Halas, N. J. Chem. Phys. Lett. 1998, 288, 243.

(2) Stober, W.; Fink, A.; Bohn, E. J. Colloid Interface Sci. 1968, 26, 62.

(3) Duff, D. G.; Baiker, A.; Edwards, P. P. Langmuir 1993, 9, 2301.

(4) Tam, F.; Moran, C. E.; Halas, N. J. J. Phys. Chem. B. 2004, 108, 17290. 